\title{
Effects of Adiposity and Prader-Willi Syndrome on Postexercise Heart Rate Recovery
}

\author{
Diobel M. Castner, ${ }^{1}$ Daniela A. Rubin, ${ }^{1}$ Daniel A. Judelson, ${ }^{1}$ and Andrea M. Haqq ${ }^{2}$ \\ ${ }^{1}$ Department of Kinesiology, California State University, Fullerton, 800 North State College Boulevard, KHS-236, \\ Fullerton, CA 92831, USA \\ ${ }^{2}$ Department of Pediatrics, University of Alberta, Edmonton, AB, Canada T6G 2R7
}

Correspondence should be addressed to Diobel M. Castner; dcastner@fullerton.edu and Daniela A. Rubin; drubin@fullerton.edu

Received 26 October 2012; Revised 21 April 2013; Accepted 23 April 2013

Academic Editor: Dénés V. Molnár

Copyright (C) 2013 Diobel M. Castner et al. This is an open access article distributed under the Creative Commons Attribution License, which permits unrestricted use, distribution, and reproduction in any medium, provided the original work is properly cited.

Heart rate recovery (HRR) is an indicator of all-cause mortality in children and adults. We aimed to determine the effect of adiposity and Prader-Willi Syndrome (PWS), a congenital form of obesity, on HRR. Sixteen children of normal weight (NW = body fat $\% \leq 85$ th percentile, $9.4 \pm 1.1 \mathrm{y}), 18$ children with obesity $(\mathrm{OB}=$ body fat $\%>95$ th percentile, $9.3 \pm 1.1 \mathrm{y}$ ), and 11 PWS youth (regardless of body fat \%; $11.4 \pm 2.5 \mathrm{y}$ ) completed peak and submaximal bike tests on separate visits. HRR was recorded one minute following peak and submaximal exercises. All groups displayed similar HRR from peak exercise, while NW ( $54 \pm 16$ beats) and OB (50 \pm 12 beats) exhibited a significantly faster HRR from submaximal exercise than PWS ( $37 \pm 14$ beats). These data suggest that excess adiposity does not influence HRR in children, but other factors such as low cardiovascular fitness and/or autonomic dysfunction might be more influential.

\section{Introduction}

Heart rate recovery (HRR) is the rate at which heart rate declines from the end of exercise to a predetermined time point during recovery, typically one minute after exercise [1, 2]. HRR depends on an immediate parasympathetic nervous system reactivation followed by sympathetic activity withdrawal [3]. After submaximal exercise, the withdrawal from sympathetic activity typically takes place 30 seconds into recovery $[2,4]$. In contrast, increased sympathetic activity following peak intensity exercise may continue for approximately one to three minutes, delaying $\operatorname{HRR}[2,5,6]$. A slower HRR has been related with lower cardiovascular fitness, thereby indicating an increased risk of cardiovascular disease [7]. Therefore, HRR has been suggested as a noninvasive method for cardiovascular risk assessment in both children and adults $[3,8]$.

Body fat is positively associated with risk for cardiovascular disease in children [9] and adults [10]. As HRR is an indirect measure of cardiovascular disease risk, studies in adults have shown that indices of obesity are strong predictors of HRR following exercise [3, 11]. Dimkpa and Oji showed that in healthy adults, obesity surrogates negatively correlated with HRR following submaximal intensity exercise [11]. Similarly, Singh and colleagues demonstrated that overweight youth experienced a slower one-minute postexercise HRR than healthy weight youth after a maximal protocol followed by one-minute cool-down period [8]. Moreover, Dangardt and colleagues suggested that children with obesity displayed a significantly lower vagal activity at rest compared to overweight and lean controls [12]. Though these studies related body fat and HRR, no research has investigated HRR from aerobic exercise in children with excessive adiposity (i.e., those classified as obese, not overweight) compared to lean controls.

In addition, measuring HRR in children predisposed to excessive adiposity (e.g., individuals with Prader-Willi Syndrome) can also reveal particular mechanisms that affect postexercise HRR response. Prader-Willi Syndrome (PWS) is the best characterized genetic cause of childhood obesity [13]. PWS is characterized by hypotonia, hyperphagia, abnormally high adiposity, hypogonadism, and lack of normal growth 
hormone production [14]. Past work investigating autonomic nervous system (ANS) function in youth with PWS presents equivocal results [15-18]; therefore, those with PWS are a particularly unique comparison group to nonsyndromal children as HRR may be related to congenital adiposity and/or syndrome-related ANS dysfunction.

Stinted HRR immediately following peak exercise reveals deficits in parasympathetic reactivation $[2,5,6]$, while stinted HRR from submaximal exercise reveals deficiencies in either parasympathetic reactivation or sympathetic withdrawal [2, 4]. Thus, studying HRR response from peak and submaximal exercise provides information about different ANS regulatory mechanisms. This study investigated whether adiposity and PWS influenced postexercise HRR from two different exercise intensities.

\section{Methods}

2.1. Participants. Sixteen (7 M and $9 \mathrm{~F}$ ) children of normal weight (NW $=$ body fat percentage $\leq 85$ th percentile for age and sex; BMI z-score: $-0.1 \pm 0.6$; age: $9.4 \pm 1.1 \mathrm{y}), 18(12 \mathrm{M}$ and $6 \mathrm{~F})$ children with obesity $(\mathrm{OB}=$ body fat percentage $>95$ th percentile for age and sex; BMI z-score: $2.0 \pm 0.5$; age: $9.3 \pm$ $1.1 \mathrm{y}$ ) [19], and 11 ( $8 \mathrm{M}$ and $3 \mathrm{~F}$ ) youth diagnosed with PWS (BMI z-score: $1.7 \pm 0.7$; age: $11.4 \pm 2.5 \mathrm{y}$ ) participated in this study. This study was approved by the Institutional Review Boards from California State University, Fullerton, Children's Hospital of Orange County, and the United States Army Medical Research and Materiel Command. Written informed assent and consent were obtained from all participants and parents prior to participation. Children with diabetes mellitus type 2, confirmed pregnancy, or those unable to participate in moderate to vigorous physical activity were excluded from participation.

2.2. Experimental Design. Participants completed two visits separated by two to 14 days. During visit one, all participants were measured for anthropometrics, body composition, resting heart rate (HR), and blood pressure (BP); subjects then completed a graded exercise test. During visit two, participants performed a discontinuous submaximal test. Participants were instructed not to engage in physical activity (i.e., sports games, bike rides, physical education, etc.) for a minimum of 24 hours before exercise testing. In addition, participants were provided with a standardized breakfast that contained no caffeine to consume two hours prior to visit arrival. This study was part of a larger research effort devoted to examining the physiological and hormonal responses to exercise in youth with PWS.

\subsection{Visit One}

2.3.1. Medical Screening. Parents of participants completed a medical history questionnaire regarding their child's health and participation in moderate to vigorous physical activity. Children without PWS completed the Pubertal Developmental Scale [20] to assess pubertal status. PWS youth underwent a full health screening by a physician to determine contraindications for exercise testing and participation in the study, as well as Tanner stage determined by breast, genital, and pubic hair development.

2.3.2. Anthropometric and Physiologic Measurements. Participants removed shoes before all measurements. Body mass was measured using a digital scale (ES200L, Ohaus, Pinewood, NJ) while participants wore a t-shirt and shorts. Height was measured after participants inhaled using a wallmounted stadiometer (Seca, ON, Canada). Waist circumference was measured following NHANES guidelines at the top of the participant's iliac crest at the end of exhalation [21]. Total body fat percentage was determined using a whole body dual energy X-ray absorptiometry (DXA) scan (GE Healthcare, GE Lunar Corp., Madison, WI). Female participants who had their first menses were required to complete a pregnancy test prior to completing the DXA scan. Resting HR, measured via telemetry (Polar USA, Lake Success, NY), and resting BP, measured via aneroid sphygmomanometer (Diagnostix 752, American Diagnostic Corporation, Hauppage, NY), were recorded following five minutes of seated rest [22].

2.3.3. Peak Graded Exercise Test. Participants completed the McMaster protocol, a cycling protocol tailored to height and sex [23], on a cycle ergometer (Corival Pediatric, Lode B.V., the Netherlands; Technogym Bike Med, Technogym USA Corp., Seattle, WA) suited to the child's stature. The test consisted of two-minute stages at incremental workloads until the participant reached volitional exhaustion, failed to sustain the desired workload [24], requested to stop, stood up on the bike pedals, or experienced fatigue-related symptoms [25].

Relative peak power output was computed by dividing the test termination load by the participant's lean body mass obtained from the DXA scan. BP was measured at rest and the end of exercise. HR was recorded at test termination and one minute following exercise. HRR was determined by computing a HRR value (HRRV) calculated as the difference between test termination $\mathrm{HR}$ and $\mathrm{HR}$ recorded after one minute after exercise [1]. One-minute postexercise HRR is the most commonly used methodology to assess recovery heart rate in children $[1-3,6,8]$.

2.4. Visit Two. Participants completed a discontinuous submaximal test consisting of ten two-minute cycling intervals each separated by one minute of rest. The resistance setting was based on the HR obtained during the McMaster protocol and chosen to elicit a $\mathrm{HR} \geq 160 \mathrm{bpm}$ throughout. This intensity was chosen based on a previous study that used a discontinuous protocol in youth of normal weight and obesity to assess counterregulatory hormonal responses to acute exercise [26]. $\mathrm{BP}$ and HR were measured and recorded using the same procedures as visit one.

2.5. Data Processing and Analysis. One-way analysis of variance (ANOVA) tests were initially conducted to determine 
TABle 1: Participant demographics and characteristics, presented as frequencies and mean \pm SD.

\begin{tabular}{|c|c|c|c|}
\hline & NW & $\mathrm{OB}$ & PWS \\
\hline Frequency & 16 & 18 & 11 \\
\hline Male/female & $7 / 9$ & $12 / 6$ & $8 / 3$ \\
\hline Age (y) & $9.4 \pm 1.1^{*}$ & $9.3 \pm 1.1^{\ddagger}$ & $11.4 \pm 2.5$ \\
\hline \multicolumn{4}{|l|}{ Pubertal stage (Tanner) } \\
\hline I & 9 & 9 & 3 \\
\hline II & 4 & 5 & 2 \\
\hline III & 3 & 3 & 2 \\
\hline IV & 0 & 0 & 2 \\
\hline $\mathrm{V}$ & 0 & 0 & 1 \\
\hline Body mass (kg) & $32.76 \pm 5.58$ & $53.18 \pm 13.31^{*}$ & $57.70 \pm 22.67^{*}$ \\
\hline Body mass index $z$-score & $-0.1 \pm 0.6$ & $2.0 \pm 0.5^{*}$ & $1.7 \pm 0.7^{*}$ \\
\hline Waist circumference $(\mathrm{cm})$ & $60.7 \pm 4.4$ & $85.3 \pm 11.9^{*}$ & $84.1 \pm 17.8^{*}$ \\
\hline Trunk fat (\%) & $16.7 \pm 5.1$ & $42.9 \pm 7.8^{*}$ & $43.5 \pm 8.4^{*}$ \\
\hline Fat mass (\%) & $18.7 \pm 4.6$ & $41.2 \pm 7.5^{*}$ & $43.4 \pm 8.2^{*}$ \\
\hline Lean mass (\%) & $78.1 \pm 4.3$ & $57.0 \pm 7.2^{*}$ & $54.8 \pm 7.8^{*}$ \\
\hline Lean mass (kg) & $25.32 \pm 3.91$ & $29.31 \pm 4.42$ & $30.91 \pm 11.92$ \\
\hline Resting heart rate (bpm) & $86 \pm 10$ & $80 \pm 9$ & $81 \pm 13$ \\
\hline Resting SBP (mm Hg) & $99 \pm 9$ & $106 \pm 11$ & $113 \pm 12^{*}$ \\
\hline Resting DBP (mm Hg) & $60 \pm 12$ & $69 \pm 11^{*}$ & $68 \pm 6$ \\
\hline
\end{tabular}

Values are significant at $P<0.050 ;{ }^{*}$ different than NW; ${ }^{\ddagger}$ different than PWS. Pubertal status was not obtained from one OB and one PWS.

group differences for participant characteristics and all exercise responses. HRRV was used to compare HRR among groups for both exercise intensities. ANOVAs were then conducted to determine group differences for HRRV for each exercise intensity. In case of significant group differences, Tukey's post hoc tests were used to determine pair-wise differences. Significance level for all statistical analyses was set at $P<0.050$. IBM SPSS Statistics 19.0 for Windows (SPSS, Inc., Chicago, IL) was used for the statistical analysis.

\section{Results}

No sex differences were found between and within groups for HRR; therefore, all children were analyzed together. As expected, OB and PWS displayed a significantly greater total body mass, waist circumference, trunk body fat percentage, and total body fat percentage compared to NW. In contrast, NW had significantly greater lean mass percentage than their counterparts; there was no difference in lean mass percentage between OB and PWS (Table 1). All groups had a similar absolute peak workload, peak SBP, and peak DBP, while youth with PWS exhibited a lower relative peak power output (expressed per $\mathrm{kg}$ of lean body mass) and peak HR than NW or OB (see Table 2), who had similar relative peak workloads and HR. Absolute submaximal workload was also similar amongst groups; however, PWS had a lower relative power output than NW only during submaximal exercise (see Table 3)-OB children were not significantly different from either PWS or NW. When expressed as a percentage of peak relative workload, all groups worked at a similar aerobic effort during submaximal exercise (Table 3). All groups had significantly different mean submaximal exercise
HR responses, with NW having the highest and PWS having the lowest (see Table 3). When expressed as a percentage of peak HR, the HR response during submaximal exercise was significantly lower in OB compared to NW; PWS was similar to both groups (Table 3 ). OB also had a significantly higher SBP in response to submaximal exercise compared to NW, and PWS had a similar response to both groups. All groups had a similar submaximal exercise DBP.

There were no significant group differences for HRRV from peak exercise. For submaximal intensity exercise, youth with PWS had a significantly lower HRRV compared to both NW and OB, who had similar HRRV (Figure 1).

\section{Discussion}

The results of this study showed that the decline in heart rate following exercise was not dependent on excessive adiposity. All groups had similar HRR in response to peak exercise. The only difference observed was in youth with PWS who displayed a significantly slower HRR following submaximal intensity exercise compared to other children. Two possible mechanisms explain the lower HRR following submaximal exercise in PWS: cardiovascular fitness and/or autonomic abnormality.

Children of normal weight and those with obesity but without PWS had similar recovery responses following both peak and submaximal aerobic exercises. Therefore, the results of this study contradicted the results of an earlier study by showing that increased adiposity did not affect postexercise HRR in children with nonsyndromic obesity [8]. However, there was a key methodological difference between the previous study [8] and the present study. The present study 
TABLE 2: Responses to peak intensity exercise, presented as mean \pm SD.

\begin{tabular}{lccc}
\hline & NW & OB & PWS \\
\hline Absolute peak power output (W) & $96.7 \pm 16.9$ & $105.9 \pm 21.4$ & $94.6 \pm 55.7$ \\
Relative peak power output $\left(\mathrm{W} \cdot \mathrm{kg} \mathrm{LBM}^{-1}\right)$ & $3.8 \pm 0.5^{\ddagger}$ & $3.6 \pm 0.7^{\ddagger}$ & $2.9 \pm 0.7$ \\
Peak heart rate (bpm) & $186 \pm 12^{\ddagger}$ & $190 \pm 14^{\ddagger}$ & $167 \pm 18$ \\
Peak SBP (mm Hg) & $129 \pm 19$ & $142 \pm 18$ & $126 \pm 20$ \\
Peak DBP (mm Hg) & $63 \pm 9$ & $67 \pm 13$ & $69 \pm 9$ \\
\hline
\end{tabular}

Values are significant at $P<0.050 ;{ }^{\ddagger}$ different than PWS.

TABLE 3: Responses to submaximal intensity exercise, presented as mean \pm SD.

\begin{tabular}{lccc}
\hline & NW & OB & PWS \\
\hline Absolute submaximal power output (W) & $67.5 \pm 14.0$ & $69.0 \pm 14.8$ & $64.8 \pm 31.3$ \\
Relative submaximal power output (W·kg LBM & & \\
Percentage of peak relative workload (\%) & $2.7 \pm 0.4$ & $2.4 \pm 0.4$ & $7.1 \pm 0.3^{*}$ \\
Submaximal exercise heart rate (bpm) & $70.9 \pm 15.0$ & $65.9 \pm 10.5$ & $71.5 \pm 10.5$ \\
Percentage of peak heart rate (\%) & $170 \pm 8^{\ddagger}$ & $161 \pm 9^{*}$ & $148 \pm 15^{* \dagger}$ \\
Submaximal SBP (mm Hg) & $91.6 \pm 5.5$ & $85.1 \pm 6.1^{*}$ & $88.7 \pm 5.4$ \\
Submaximal DBP (mm Hg) & $122 \pm 12$ & $136 \pm 12^{*}$ & $129 \pm 20$ \\
\hline
\end{tabular}

Values are significant at $P<0.050 ;{ }^{*}$ different than NW; ${ }^{\dagger}$ different than $\mathrm{OB} ;{ }^{\ddagger}$ different than PWS.
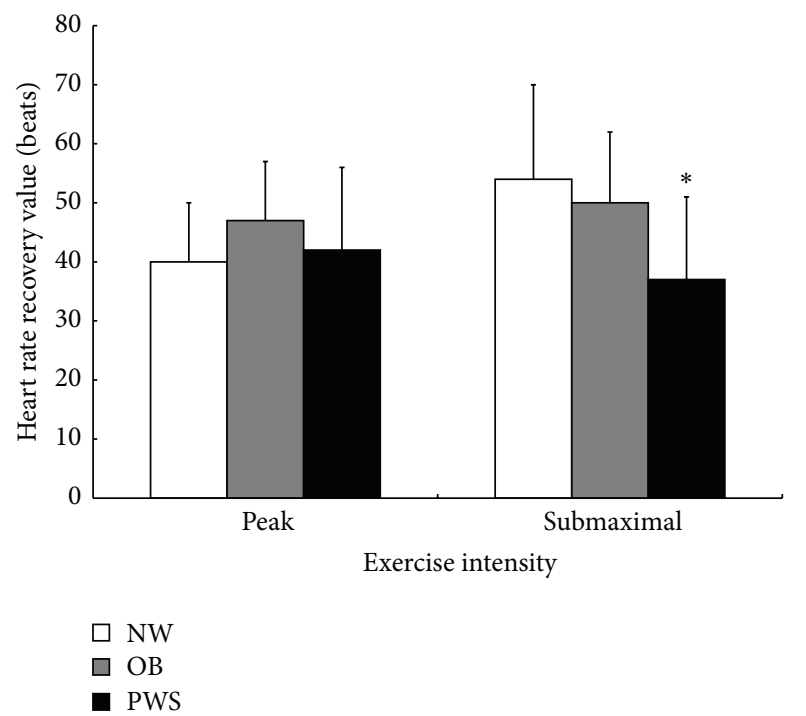

FIGURE 1: Postexercise HRRV (beats) by group and exercise intensity, presented as mean $\pm \mathrm{SD} ;{ }^{*} \mathrm{P}<0.050$.

measured passive (i.e., no cool down, seated) HRR immediately following test termination. By doing so, postexercise HRR was not affected by continued, voluntary sympathetic activation (which occurs during active cool down) and better isolated true recovery rate.

Another factor that could have influenced the HRR results of this study is cardiovascular fitness. Higher levels of cardiovascular fitness have been positively associated with a faster HRR following exercise [27-31]. Adults with high levels of physical activity have shown to either sustain or improve HRR over 20 years compared to adults with low levels of physical activity [28]. In addition, HRR improved after exercise training and a hypocaloric diet in obese children
[31]. The fact that cardiovascular fitness is related to HRR may partially explain the results of this study. Both participants with obesity and those of normal weight were similarly fit (i.e., peak $\mathrm{W} \cdot \mathrm{kg} \mathrm{LBM}{ }^{-1}$, peak $\mathrm{HR}$, and peak estimated [23] $\mathrm{VO}_{2 \text { peak }}\left(13.9 \pm 1.0\right.$ versus $\left.\left.13.6 \pm 1.2 \mathrm{~mL} \cdot \mathrm{kg} \mathrm{LBM}^{-1} \cdot \mathrm{min}\right)\right)$. In contrast, those with PWS were significantly less fit compared to children without PWS (i.e., lower peak HR, lower W·kg $\mathrm{LBM}^{-1}$ and lower estimated [23] $\mathrm{VO}_{2 \text { peak }}(12.4 \pm 1.3 \mathrm{~mL} \cdot \mathrm{kg}$ $\left.\mathrm{LBM}^{-1} \cdot \mathrm{min}\right)$ ). Previous work showed poor cardiovascular fitness and low physical activity level in PWS [32, 33]. Poor cardiovascular fitness in PWS might be best explained by physiological characteristics inherent to the syndrome including hypotonia [32], reduced knee flexor and extensor muscle strength [34], and/or muscle fiber size deficiency and atrophy [35]. Therefore, the lower HRR after submaximal exercise in those with PWS compared to the other children possibly indicates that the low cardiovascular fitness in those with PWS was related to the slow $\operatorname{HRR}[28,31,36]$.

In addition, it is possible that HRR in PWS may have also been influenced by altered ANS function. A review by Haqq et al. suggests that in obesity there is an increased sympathetic activity as well as reduced parasympathetic activity [37]. This mechanism is not so clear in PWS, although it appears that PWS presents similarities with an autonomic disorder called familial dysautonomia where more sympathetic neurons are affected than parasympathetic neurons [37]. Richer and colleagues investigated a small cohort of children with PWS and controls to determine possible differences in ANS function. Postganglionic sympathetic function was evaluated through Quantitative Sudomotor Axon Reflex testing (sweat volume), cardiovagal testing (HR response to deep breathing), and pupillary response (HR and BP responses and HR variability spectral analysis to head-up tilt). Children with PWS exhibited a trend towards lower total sweat volume, a smaller HR increase with head tilt-up, and lower low frequency power 
HR variability at rest and with head-up tilt compared to the controls indicating possible impaired sympathetic function [15].

The results of the present study showed a lower peak HR in PWS perhaps related to lower muscular work capacity or sympathetic stimulation. However, the lower HRR following submaximal exercise in those with PWS compared to nonsyndromal children may indicate a delayed sympathetic withdrawal and impaired function as suggested by Richer and colleagues [15]. In terms of peak exercise responses, it is possible that the chosen protocol limited the capacity to fully assess ANS function, and therefore no differences among groups were obtained. Past studies suggested that after peak intensity exercise HRR is delayed due to sympathetic activity lasting one to three minutes into the recovery period. The present study measured HRR only during the first minute after exercise, and this is a limitation of the study design and results $[2,5]$.

In addition, the participants with PWS were significantly older than nonsyndromal obese controls, which might explain the deterioration in ANS function [38]. Seven youth with PWS presented Tanner stages I-III, while two youth presented stage IV and one stage $\mathrm{V}$. In comparison, all nonsyndromal children presented Tanner stages I through III based on their self-report. Follow-up analyses were done to determine differences among groups in HRR including only participants with Tanner stages I through III. Youth with PWS $(n=7)$ still presented a significantly slower HRR following submaximal intensity exercise compared to NW $(n=16)$. However, OB $(n=17)$ responded similarly to both groups following submaximal exercise. Similar to the previous analyses, no group differences were observed following maximal intensity exercise. It is possible that those youth with PWS in later stages of puberty experienced more ANS deterioration, exacerbating the HRR differences with the nonsyndromal obese controls. Regardless, youth with PWS still showed a delayed recovery from submaximal intensity exercise compared with normal weight controls, indicating possible ANS dysfunction. It is necessary for future studies to account for pubertal status as a screening criterion to better assess whether the differences in HRR are related to ANS function. Lastly, recording blood pressure at the end of the recovery period is another measurement that could have helped assess the ANS function in response to exercise. Overall, the results of this study suggest that ANS dysfunction in youth with PWS could influence HRR following submaximal intensity exercise.

Having participants with PWS complete these protocols presented barriers. Most of these limitations were related to the physiological (lower than normal muscle force capacity, lack of stamina, and lower than normal motor proficiency), psychological (mild to severe mental retardation and mental rigidity), and behavioral (attention deficit, temper tantrums, and inability to accept change) characteristics of PWS [13, 14, 32, 33, 39]. Even with these difficulties, those with PWS were able to complete a graded peak exercise protocol. It was verified that the submaximal effort was similar between those with and without PWS by calculating the percentage of relative power output and heart rate compared to peak effort. Therefore, the present study results were not affected by the exercise protocols. The present study lacked comparison groups with low and high cardiovascular fitnesses, a comparison that would have isolated the effect of cardiovascular fitness on HRR. However, a study with such research design may not be feasible given that inherently PWS presents poor cardiovascular fitness.

\section{Conclusion}

As demonstrated by the similar recovery rate in children of normal weight and those with obesity, adiposity did not influence HRR following peak or submaximal exercise. Youth with PWS showed a low cardiovascular recovery capacity, suggesting that poor cardiovascular fitness and/or an altered ANS function may impact HRR.

\section{Conflict of Interests}

The authors claim no conflict of interests for this paper.

\section{Acknowledgments}

The authors would like to thank the participants and their families, Dr. Susan Clark, and the staff at the Children's Hospital of Orange County. This study was supported by the US Army Medical Research and Materiel Command Award W81XWH-08-1-0025. Dr. Haqq received funding from the Canadian Institutes of Health Research (CIHR), Foundation for Prader-Willi Research (FPWR), Women \& Children's Health Research Institute, and the Alberta Diabetes Institute at University of Alberta.

\section{References}

[1] A. D. Mahon, C. S. Anderson, M. J. Hipp, and K. A. Hunt, "Heart rate recovery from submaximal exercise in boys and girls," Medicine and Science in Sports and Exercise, vol. 35, no. 12, pp. 2093-2097, 2003.

[2] G. L. Pierpont, D. R. Stolpman, and C. C. Gornick, "Heart rate recovery post-exercise as an index of parasympathetic activity," Journal of the Autonomic Nervous System, vol. 80, no. 3, pp. 169$174,2000$.

[3] C. R. Cole, E. H. Blackstone, F. J. Pashkow, C. E. Snader, and M. S. Lauer, "Heart-rate recovery immediately after exercise as a predictor of mortality," The New England Journal of Medicine, vol. 341, no. 18, pp. 1351-1357, 1999.

[4] H. Ohuchi, H. Suzuki, K. Yasuda, Y. Arakaki, S. Echigo, and T. Kamiya, "Heart rate recovery after exercise and cardiac autonomic nervous activity in children," Pediatric Research, vol. 47, no. 3, pp. 329-335, 2000.

[5] N. Nagai and T. Moritani, "Effect of physical activity on autonomic nervous system function in lean and obese children," International Journal of Obesity, vol. 28, no. 1, pp. 27-33, 2004.

[6] E. Baraldi, D. M. Cooper, S. Zanconato, and Y. Armon, "Heart rate recovery from 1 minute of exercise in children and adults," Pediatric Research, vol. 29, no. 6, pp. 575-579, 1991.

[7] M. Buchheit, G. P. Millet, A. Parisy, S. Pourchez, P. B. Laursen, and S. Ahmaidi, "Supramaximal training and postexercise parasympathetic reactivation in adolescents," Medicine 
and Science in Sports and Exercise, vol. 40, no. 2, pp. 362-371, 2008.

[8] T. P. Singh, J. Rhodes, and K. Gauvreau, "Determinants of heart rate recovery following exercise in children," Medicine and Science in Sports and Exercise, vol. 40, no. 4, pp. 601-605, 2008.

[9] M. Dencker, P. Wollmer, M. K. Karlsson, C. Lindén, L. B. Andersen, and O. Thorsson, "Body fat, abdominal fat and body fat distribution related to cardiovascular risk factors in prepubertal children," Acta Paediatrica, vol. 101, no. 8, pp. 852857, 2012.

[10] P. Poirier, T. D. Giles, G. A. Bray et al., "Obesity and cardiovascular disease: pathophysiology, evaluation, and effect of weight loss: an update of the 1997 American Heart Association Scientific Statement on obesity and heart disease from the Obesity Committee of the Council on Nutrition, Physical Activity, and Metabolism," Circulation, vol. 113, no. 6, pp. 898918, 2006.

[11] U. Dimkpa and J. O. Oji, "Association of heart rate recovery after exercise with indices of obesity in healthy, non-obese adults," European Journal of Applied Physiology, vol. 108, no. 4, pp. 695699, 2010.

[12] F. Dangardt, R. Volkmann, Y. Chen, W. Osika, S. Mårild, and P. Friberg, "Reduced cardiac vagal activity in obese children and adolescents," Clinical Physiology and Functional Imaging, vol. 31, no. 2, pp. 108-113, 2011.

[13] M. G. Butler, J. M. Hanchett, and T. Thompson, "Clinical findings and natural history of Prader-Willi syndrome," in Management of Prader-Willi Syndrome, M. G. Butler, P. D. K. Lee, and B. Y. Whitman, Eds., pp. 3-48, Springer, New York, NY, USA, 3rd edition, 2006.

[14] V. A. Holm, S. B. Cassidy, M. G. Butler et al., "Prader-Willi syndrome: consensus diagnostic criteria," Pediatrics, vol. 91, no. 2, pp. 398-402, 1993.

[15] L. P. Richer, D. S. DeLorey, A. M. Sharma et al., "Autonomic vervous system (ANS) dysfunction in PWS and childhood obesity: preliminary findings," in Proceedings of the 31st PraderWilli Syndrome Association (USA) Annual National Conference, Orlando, FL, USA, 2011.

[16] C. K. Wade, R. E. de Meersman, M. Angulo, J. S. Lieberman, and J. A. Downey, "Prader-Willi syndrome fails to alter cardiac autonomic modulation," Clinical Autonomic Research, vol. 10, no. 4, pp. 203-206, 2000.

[17] S. Patel, J. A. Harmer, G. Loughnan, M. R. Skilton, K. Steinbeck, and D. S. Celermajer, "Characteristics of cardiac and vascular structure and function in Prader-Willi syndrome," Clinical Endocrinology, vol. 66, no. 6, pp. 771-777, 2007.

[18] F. J. DiMario Jr., B. Dunham, J. A. Burleson, J. Moskovitz, and S. B. Cassidy, "An evaluation of autonomic nervous system function in patients with Prader-Willi syndrome," Pediatrics, vol. 93, no. 1, pp. 76-81, 1994.

[19] H. D. McCarthy, T. J. Cole, T. Fry, S. A. Jebb, and A. M. Prentice, "Body fat reference curves for children," International Journal of Obesity, vol. 30, no. 4, pp. 598-602, 2006.

[20] A. C. Petersen, L. Crockett, M. Richards, and A. Boxer, "A selfreport measure of pubertal status: reliability, validity, and initial norms," Journal of Youth and Adolescence, vol. 17, no. 2, pp. 117133, 1988.

[21] Centers for Disease Control, National Health and Nutrition Examination Survey (NHANES): Anthropometry Procedures Manual, N.C.F.H. Statistics, Atlanta, Ga, USA, 2007.

[22] American Academy of Pediatrics, "The fourth report on the diagnosis, evaluation, and treatment of high blood pressure in children and adolescents," Pediatrics, vol. 114, no. 2 supplement 4th Report, pp. 555-576, 2004.

[23] V. H. Heyward, Advanced Fitness Assessment and Exercise Prescription, Human Kinetics, Champaign, IL, USA, 6th edition, 2010.

[24] O. Bar-Or and T. Rowland, "Procedures for exercise testing in children," in Pedaitric Exercise Medicine: From Physiologic Principles To Health Care Application, pp. 345-365, Human Kinetics, Champaign, IL, USA, 2004.

[25] S. M. Paridon, B. S. Alpert, S. R. Boas et al., "Clinical stress testing in the pediatric age group: a statement from the American Heart Association council on cardiovascular disease in the young, committee on atherosclerosis, hypertension, and obesity in youth," Circulation, vol. 113, no. 15, pp. 1905-1920, 2006.

[26] A. Eliakim, D. Nemet, F. Zaldivar et al., "Reduced exerciseassociated response of the GH-IGF-I axis and catecholamines in obese children and adolescents," Journal of Applied Physiology, vol. 100, no. 5, pp. 1630-1637, 2006.

[27] J. C. Sieverdes, X. Sui, and S. N. Blair, "Associations between physical activity and submaximal cardiorespiratory and pulmonary responses in men," Journal of Sports Medicine \& Doping Studies, vol. 1, no. 1, 2011.

[28] M. R. Carnethon, B. Sternfeld, K. Liu et al., "Correlates of heart rate recovery over 20 years in a healthy population sample," Medicine and Science in Sports and Exercise, vol. 44, no. 2, pp. 273-279, 2012.

[29] C. M. Lee and A. Mendoza, "Dissociation of heart rate variability and heart rate recovery in well-trained athletes," The European Journal of Applied Physiology, vol. 112, no. 7, pp. 27572766, 2012.

[30] K. Imai, H. Sato, M. Hori et al., "Vagally mediated heart rate recovery after exercise is accelerated in athletes but blunted in patients with chronic heart failure," Journal of the American College of Cardiology, vol. 24, no. 6, pp. 1529-1535, 1994.

[31] D. M. Prado, A. G. Silva, I. C. Trombetta et al., "Exercise training associated with diet improves heart rate recovery and cardiac autonomic nervous system activity in obese children," International Journal of Sports Medicine, vol. 31, no. 12, pp. 860$865,2010$.

[32] M. G. Butler, M. F. Theodoro, D. C. Bittel, and J. E. Donnelly, "Energy expenditure and physical activity in Prader-Willi syndrome: comparison with obese subjects," American Journal of Medical Genetics A, vol. 143, no. 5, pp. 449-459, 2007.

[33] E. G. A. H. van Mil, K. R. Westerterp, W. J. M. Gerver, W. D. van Marken Lichtenbelt, A. D. M. Kester, and W. H. M. Saris, "Body composition in Prader-Willi syndrome compared with nonsyndromal obesityrelationship to physical activity and growth hormone function," Journal of Pediatrics, vol. 139, no. 5, pp. 708-714, 2001.

[34] P. Capodaglio, L. Vismara, F. Menegoni, G. Baccalaro, M. Galli, and G. Grugni, "Strength characterization of knee flexor and extensor muscles in Prader-Willi and obese patients," BMC Musculoskeletal Disorders, vol. 10, article 47, 2009.

[35] L. Reus, M. Zwarts, L. A. van Vlimmeren, M. A. Willemsen, B. J. Otten, and M. W. G. Nijhuis-van der Sanden, "Motor problems in Prader-Willi syndrome: a systematic review on body composition and neuromuscular functioning," Neuroscience and Biobehavioral Reviews, vol. 35, no. 3, pp. 956-969, 2011. 
[36] C. D. Lee, S. N. Blair, and A. S. Jackson, "Cardiorespiratory fitness, body composition, and all-cause and cardiovascular disease mortality in men," American Journal of Clinical Nutrition, vol. 69, no. 3, pp. 373-380, 1999.

[37] A. M. Haqq, D. S. DeLorey, A. M. Sharma et al., "Autonomic nervous system dysfunction in obesity and Prader-Willi syndrome: current evidence and implications for future obesity therapies," Clinical Obesity, vol. 1, no. 4-6, pp. 175-183, 2012.

[38] A. R. Galeev, L. N. Igisheva, and E. M. Kazin, "Heart rate variability in healthy six- to sixteen year old children," Fiziologiia cheloveka, vol. 28, no. 4, pp. 54-58, 2002.

[39] P. Burman, E. M. Ritzén, and A. C. Lindgren, "Endocrine dysfunction in Prader-Willi syndrome: a review with special reference to GH," Endocrine Reviews, vol. 22, no. 6, pp. 787-799, 2001. 


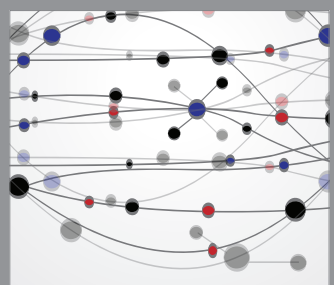

The Scientific World Journal
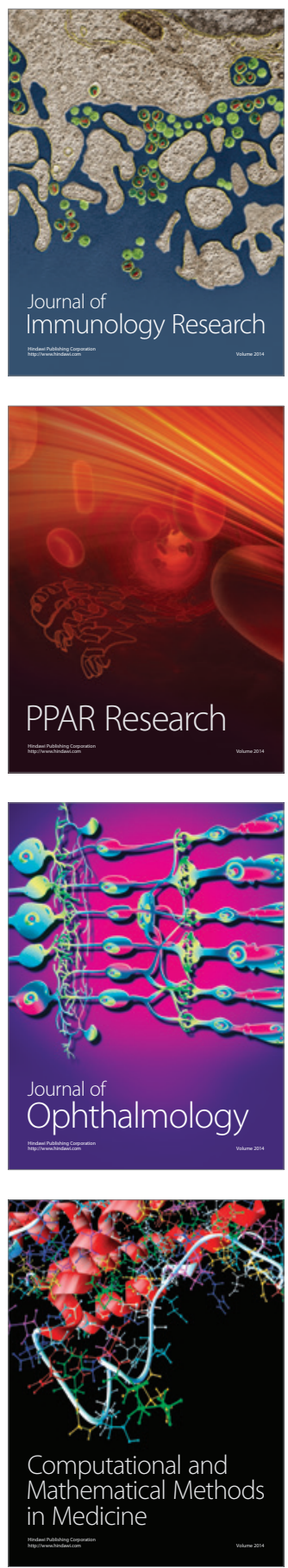

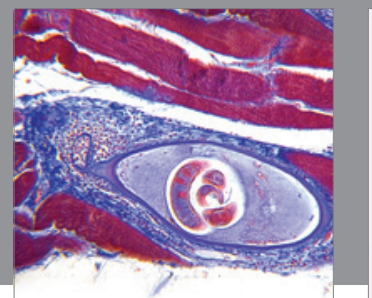

Gastroenterology

Research and Practice
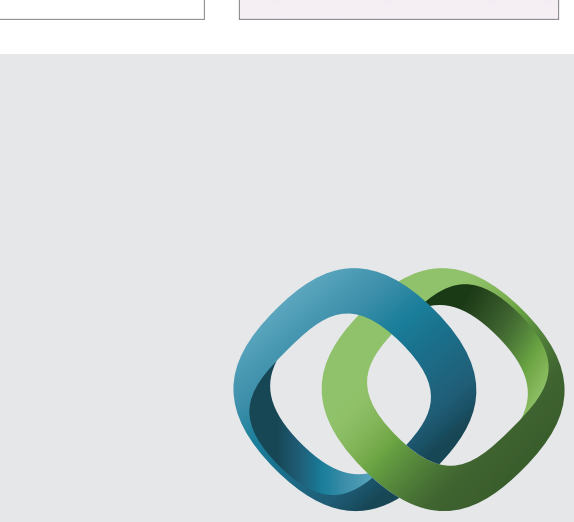

\section{Hindawi}

Submit your manuscripts at

http://www.hindawi.com
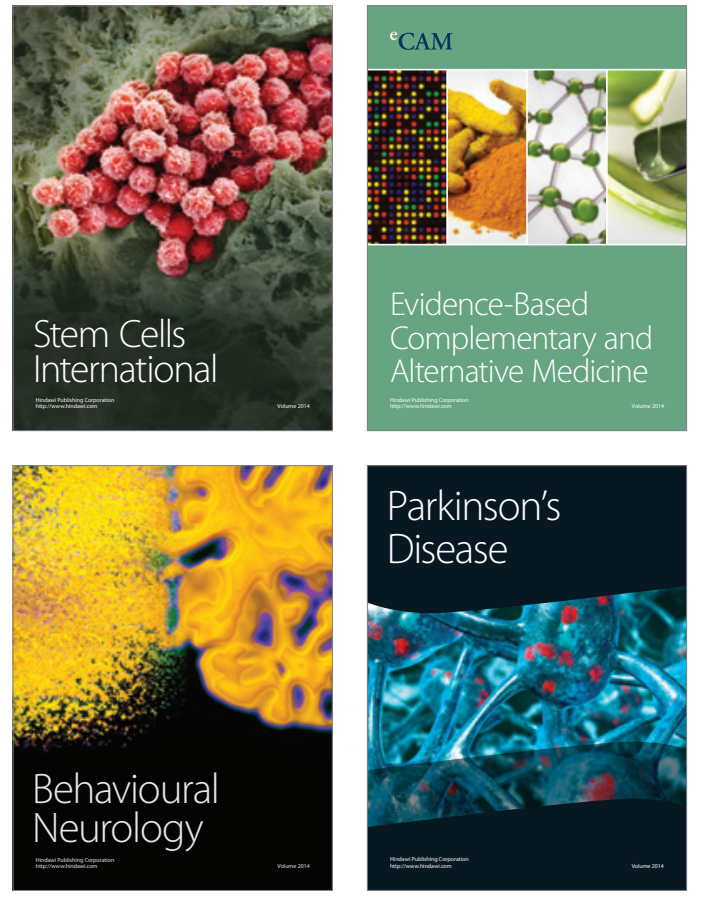
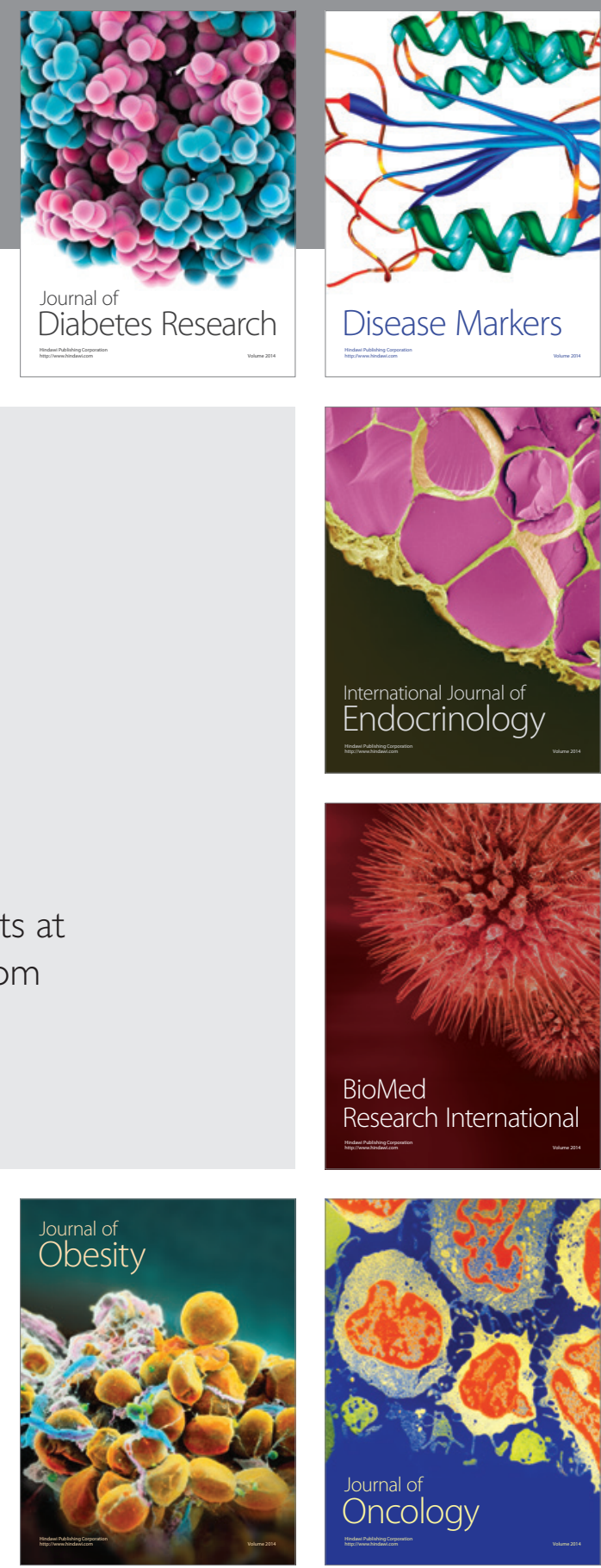

Disease Markers
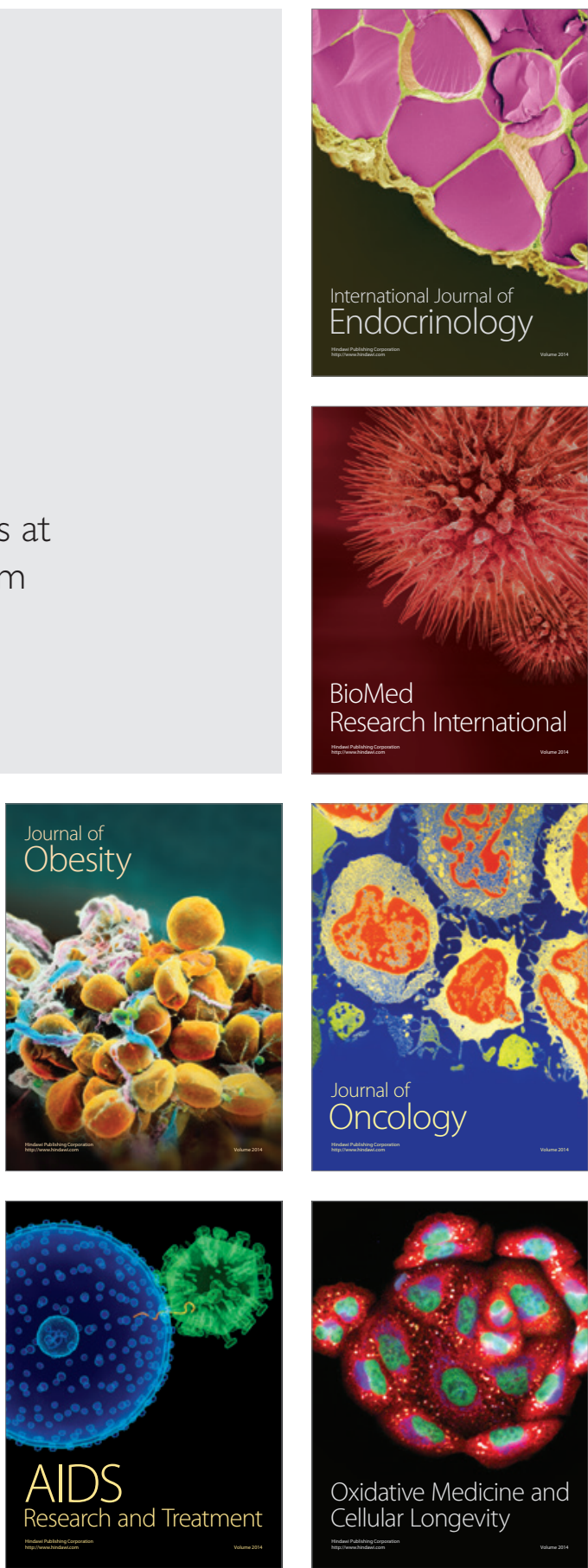